\title{
SPERT gene silencing inhibits the growth of human colon cancer xenograft tumor in nude mice via p38MAPK/HSP27 signaling pathway
}

\author{
Si-zeng $\mathrm{CHEN}^{1}$ and Long-zhi ZHENG ${ }^{2}$ \\ ${ }^{1}$ Department of Gastrointestinal Surgery, The First Affiliated Hospital of Fujian Medical University, Fuzhou 350005, Fujian, P.R. China \\ and ${ }^{2}$ Department of Gastrointestinal Surgery and Gastrointestinal Surgery Research Institute, the Affiliated Hospital of Putian University, \\ Putian 351100, Fujian, P.R. China
}

(Received 17 October 2021; and accepted 28 October 2021)

\begin{abstract}
Colorectal cancer is one of the most common gastrointestinal malignancies and is also a disease of genetic heterogeneity. Our previous studies have shown that SPERT (sprermatid-associated protein) gene may be an underlying oncogene that is associated with the progression of the disease in colorectal cancer patients, and SPERT gene silencing can inhibit the proliferation of colorectal tumor cells and promote cell apoptosis. Here, we use the stably transfected human colorectal cancer cell line RKO to construct an animal xenograft model and study the effect of SPERT gene silencing on animal xenografts. The results showed that SPERT gene silencing can inhibit tumor growth in animals. In addition, through signaling pathway analysis, we found that the p38MAPK/HSP27 signaling pathway may be the molecular mechanism by which SPERT gene silencing inhibits the growth of xenograft tumors in nude mice. Combined with previous data, SPERT gene silencing has the same inhibitory effect on tumor growth in vitro and in vivo. These data suggest that SPERT gene may be a potential target for the treatment of colorectal cancer in clinic.
\end{abstract}

\section{INTRODUCTION}

At present, cancer is still a common problem and a major burden of mankind all over the world. According to the 2018 Global Cancer Statistics Report released by the International Agency for Research on Cancer (IARC), the number of newly-increased people with colorectal cancer (CRC) in 2018 was 1.8 million, accounting for $10.2 \%$ of the total cancer population and ranking third in the global incidence of cancer, behind lung cancer and breast cancer (Bray et al. 2018). In 2018, the number of colorectal cancer deaths was 880,000 , accounting for $9.2 \%$

Address correspondence to: Dr Zheng Long-zhi, Department of Gastrointestinal Surgery, The Affiliated Hospital of Putian University, No.999, DongzhenDong Road, Licheng District, Putian 351100, Fujian Province, People's Republic of China

Tel: +86-0594-2730373, Fax: +86-0594-2293910

E-mail: shixin8217@tom.com of the total cancer incidence, ranking second in the death rate of cancer in the global population, after lung cancer. Compared with previous data (Torre et al. 2015), the proportion of colorectal cancer morbidity and mortality in the global malignant tumors has increased, and the mortality rate has jumped from fourth to second. These data suggest that reducing morbidity and mortality of colorectal cancer is still one of the arduous tasks of global cancer prevention and control.

With the rapid development of tumor genomics, the information related to human genome has been gradually expanded, and scientists' understanding of the molecular basis of cancer has been rapidly advanced, providing new opportunities for solving the practical challenges of cancer. The prognosis of colorectal cancer has been steadily improved compared with more than 10 years ago (Brenner et al. 2014). Currently, tumor genomics has identified new drug targets for genotype-specific therapy and provided strategies for validating these targets and developing 
drugs. It will provide new hope for cancer research and have a huge impact in the field of cancer medicine (Sakatani and Onyango 2003; Strausberg et al. 2004; Chery 2016; Offit 2016).

Our previous studies have showed that the expression of SPERT in cancer tissues of colorectal cancer patients is significantly higher than that in paracancerous tissues, and is related to the progression of the disease in colorectal cancer patients. It may be a potential oncogene, and its molecular mechanism may be closely related to the MAPK signaling pathway (Zheng and Chen 2018). However, how SPERT promotes the occurrence and progression of colon cancer and the specific molecular mechanisms of its role are still poorly understood. In this study, we further verified the function of the SPERT gene by constructing an animal xenograft model, and explored its molecular mechanism through signal pathway analysis. Our research aims to provide new insights into the molecular function of SPERT and its regulatory mechanism in colorectal cancer.

\section{MATERIALS AND METHODS}

Cell culture. The human CRC cell line RKO was purchased from the Cell Bank of the Chinese Academy of Sciences (Shanghai, China; cat. no. CBP60006). The cells were incubated in RPMI-1640 medium (Beyotime Institute of Biotechnology, Shanghai, China) supplemented with $10 \%$ fetal bovine serum (Ausbian, Adelaide, Australia) at $37^{\circ} \mathrm{C}$ in an atmosphere of $5 \% \mathrm{CO}_{2}$. Human CRC RKO cells stably transfected with LV-SPERT-RNAi (shSPERT group /KD group) and LV-shRNA-NC (shCtrl group / $\mathrm{NC}$ group) were successfully constructed in our laboratory.

In vivo tumorigenesis in nude mice. $\mathrm{BALB} / \mathrm{c}$ nude mice were purchased from Shanghai Lingchang Biotechnology Co., Ltd. (SCXK 2018-0003; Shanghai, China), and were bred in SPF level animal laboratory to perform experiments according to "GUIDELINES FOR TUMOR INDUCTION IN MICE AND RATS (UPDATED MAY 2013)". A total of $4 \times 10^{6}$ cells from individually constructed RKO cell lines in log-phase were resuspended in $0.2 \mathrm{~mL}$ PBS and injected into the right axillary skin of BALB/c nude mice. Tumor growth was observed every two days. When the tumor diameter exceeded $20 \mathrm{~mm}$, mice were killed according to animal welfare ethics regulations, and the volume and weight of the xenograft tumors in each group were calculated. Tumor vol- ume $=\pi / 6 \times \mathrm{L} \times \mathrm{W} \times \mathrm{W}$ ( $\mathrm{L}$ for tumor length, $\mathrm{W}$ for tumor short). The resected tumor tissues were confirmed by HE staining and Western blot. In all these animal experiments, mice were randomly assigned to the experimental group. All animal experiments have been approved by the IACUC Committee of the Affiliated Hospital of Putian College.

Histologic evaluation and SPERT detection. The excised tumor tissues were formalin-fixed, paraffinembedded, and sectioned. Hematoxylin and eosin (HE; Beyotime Biotechnology, Shanghai, China) were used to observe the histological characteristics of xenotransplanted tumors, and the expression of SPERT protein in tumor tissues was detected by Western blot.

Cell proliferation assay (Ki-67). The tissue sections were dewaxed with xylene and rehydrated with gradient ethanol, and the antigen retrieval was performed with the use of citrate buffer at $1 \mathrm{mmol} / \mathrm{L}$, $\mathrm{pH} 6.0$, at $95^{\circ} \mathrm{C}$ for $10 \mathrm{~min}$. After cooling to room temperature, $3 \%$ hydrogen peroxide was used to inactivate endogenous peroxidase and goat serum was used to block non-specific antigen, and then the slides were incubated with first antibody (rabbit anti-human Ki-67 antibody, 1:200; cat. no. ab15580; Abcam, Shanghai, China) at $4^{\circ} \mathrm{C}$ overnight. Subsequently, slides were incubated with HRP-labeled goat anti-rabbit secondary antibody, stained with 3,3-diaminobenzidine (DAB) solution, and counterstained with hematoxylin. Immunohistochemical results were evaluated by two senior pathologists under double-blind conditions. The percentage of positive cells are scored as follows: no positive cells $=0,<25 \%=1,25-50 \%=2,50-75 \%=3,>75 \%=4$. The cell staining intensity score was as follows: no staining $=0$, light yellow $=1$, yellow $=2$, and brownish yellow $=3$. The percentage score of positive cells is multiplied by the score of staining intensity to obtain a final immunoreactive score (Wang et al. 2009).

TUNEL apoptosis assay. The tissue sections were dewaxed with xylene, hydrated with gradient ethanol, and permeated with the working solution of protease K. Each section was dripped with $50 \mu \mathrm{L}$ reaction mixture $(450 \mu \mathrm{L}$ luciferin-labeled dUTP + $50 \mu \mathrm{L} \mathrm{TdT}$ ), and incubated in a dark wet box at $37^{\circ} \mathrm{C}$ for $1 \mathrm{~h}$. Then, HRP-labeled anti-fluorescein antibody (converter-POD) was added and incubated in a dark wet box at $37^{\circ} \mathrm{C}$ for $30 \mathrm{~min}$. Then, DAB was used for color development, and the slides were counterstained with hematoxylin. After dehydration, trans- 
parency and sealing, TUNEL positive cells were counted under the microscope (Yang et al. 1996).

Western blot analysis. Total protein was extracted using RIPA lysis buffer and quantified using a BCA protein assay kit (both from Beyotime Institute of Biotechnology). Total protein was then separated by $10 \%$ SDS-PAGE, and transferred to PVDF membranes (EMD Millipore, Bedford, MA, USA), which were blocked and incubated at $4{ }^{\circ} \mathrm{C}$ overnight. The blots were then incubated with mouse anti-FLAG monoclonal antibodies $(1: 2,000$ dilution; cat. no. F1804; Sigma-Aldrich Trading Co. Ltd., Shanghai, China), washed three times (10 min each) with TBST, incubated with the secondary antibody (goat anti-mouse $\mathrm{IgG}$, dilution, $1: 2,000$; cat. no. sc-2005; Santa Cruz Biotechnology, Co., Ltd., Shanghai, China) for $1 \mathrm{~h}$, and washed three times (10 min each) with TBST. The membranes were viewed with an Odyssey fluorescence imaging system (LI-COR Biosciences, Lincoln, NE, USA), and immunoreactive protein bands were visualized with the Pierce ${ }^{\mathrm{TM}} \mathrm{ECL}$ Western Blotting Substrate (Pierce, Rockford, IL, USA). ImageJ (version 1.46 release; National Institutes of Health, Bethesda, MD, USA) software was used for quantification of the western blots.

Statistical analysis. All measurement data are presented as the mean \pm standard deviation (SD). Differences in the means between groups were tested for statistical significance with the Student's $t$-test, and comparison of proportions was conducted with a Chi-square test. The association of SPERT expression with clinicopathological characteristics was examined with a Mann-Whitney $U$ test. All statistical analyses were performed using SPSS statistical software version 19.0 (SPSS, Inc., Chicago, IL, USA), and a $\mathrm{P}$-value of $<0.05$ was considered to indicate a statistically significant difference.

\section{RESULTS}

SPERT silencing inhibits tumorigenesis of colorectal cancer cells in vivo

The experimental results showed when the tumor volume was measured in the same period of time, the subcutaneous tumor volume of the control group (NC group) nude mice significantly exceeded the tumor volume of the intervention group (KD group). The tumor volume of the NC group and KD group was $1101.93 \pm 541.79 \mathrm{~mm}^{3}$ and $155.45 \pm 151.63 \mathrm{~mm}^{3}$, respectively on the day the nude mice were killed. The subcutaneous tumor volume of the intervention group was significantly smaller (Fig. 1A, B and C), and the data comparison showed statistical difference $(P<0.001)$. The tumor weight of NC group and $\mathrm{KD}$ group was $0.936 \pm 0.451 \mathrm{~g}$ and $0.151 \pm 0.151 \mathrm{~g}$, respectively. The tumor weight of the intervention group was significantly reduced (Fig. 1D), and the comparison of data was statistically significant $(P<0.001)$. The above experimental results indicated that SPERT silencing could inhibit tumor growth in nude mice.

HE staining and SPERT protein expression in subcutaneous tumor tissue of nude mice

Removed subcutaneous tumors of the two groups of animals were irregular in appearance with no envelope and showed invasive growth. The tumors in the control group were generally larger. HE staining showed significant structural heterogeneity and disorganisation of the tissues; cell size and morphology were variable, with large cell heterogeneity and pathological nuclear division with a nucleoplasmic ratio greater than $1: 1$ (Fig. 2), which was identified by experienced pathologists as consistent with the characteristics of a transplant tumor. These results indicated that the xenograft tumour model was successfully constructed. In the nude mice transplanted tumor animal model, we further detected the expression level of SPERT in tumor tissue to verify the effect of SPERT knockdown on the growth of subcutaneous tumors. Because the constructed vector contains the FLAG tag fused with SPERT, the expression level of FLAG represents the expression content of SPERT. WB results (Fig. 3) show that protein expression gray scales of SPERT in tumor tissues of NC group and KD group were 1.06365574 and 0.218258572 , respectively, with statistically significant data comparison $(P<0.01)$. The results indicated that the inhibition of tumor growth in KD group was related to SPERT knockdown.

Detection of cell proliferation and apoptosis in subcutaneous tumor tissues of nude mice (Ki67 assay and TUNEL assay)

We further used immunohistochemistry to detect the expression of Ki-67 positive cells and TUNEL assay to detect the apoptosis of tumor cells, so as to comprehensively analyze the proliferation and apoptotic activities of tumor cells in the subcutaneous tumor tissue of nude mice, and to determine the effect of SPERT knockdown on tumor growth in vivo. The results of Ki-67 assay are as follows (Fig. 4): the number of Ki-67 positive cells in the KD group was reduced; the percentage of Ki-67 positive cells was 

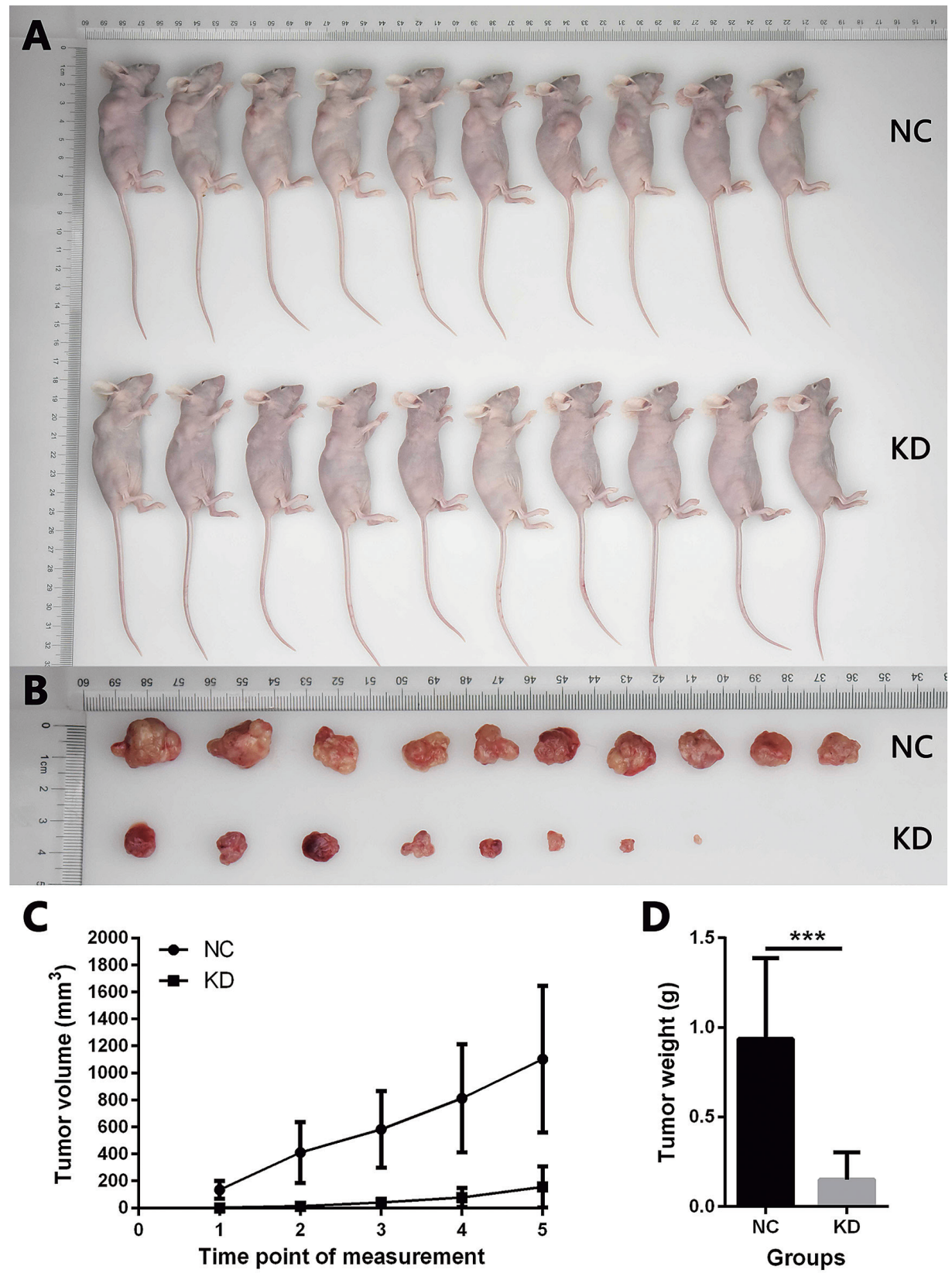

Fig. 1 The effect of SPERT silencing on subcutaneous tumor formation in nude mice. (A and B) Growth of tumors in animals after SPERT silencing in the NC and KD groups. (C) Compared with the NC group, the tumor volume in the KD group was significantly smaller $\left({ }^{* \star *} P<0.001\right)$. (D) Compared with the NC group, the tumor weight of the nude mice in the KD group was significantly reduced $\left({ }^{\star \star \star} P<0.001\right)$.

$6.5 \pm 1.2 \%$. The staining intensity of the positive cells was significantly weakened, and the nuclear staining was light yellow. The calculated immunohistochemical score of $\mathrm{Ki}-67$ in the $\mathrm{KD}$ group was 1 point (low expression). The percentage of Ki-67 positive cells in the NC group was $62.6 \pm 10.5 \%$; the staining intensity of the positive cells was strong, and the nuclear staining was brownish yellow. The immunohistochemical score of $\mathrm{Ki}-67$ in the NC group was calculated as 8 points (high expression). The positive expression of Ki-67 protein was significantly different between the two groups $(P<0.05)$, and the expression of $\mathrm{Ki}-67$ protein was low in the KD group, indicating that SPERT knockdown can reduce the proliferation activity of cells in tumor tissues. 

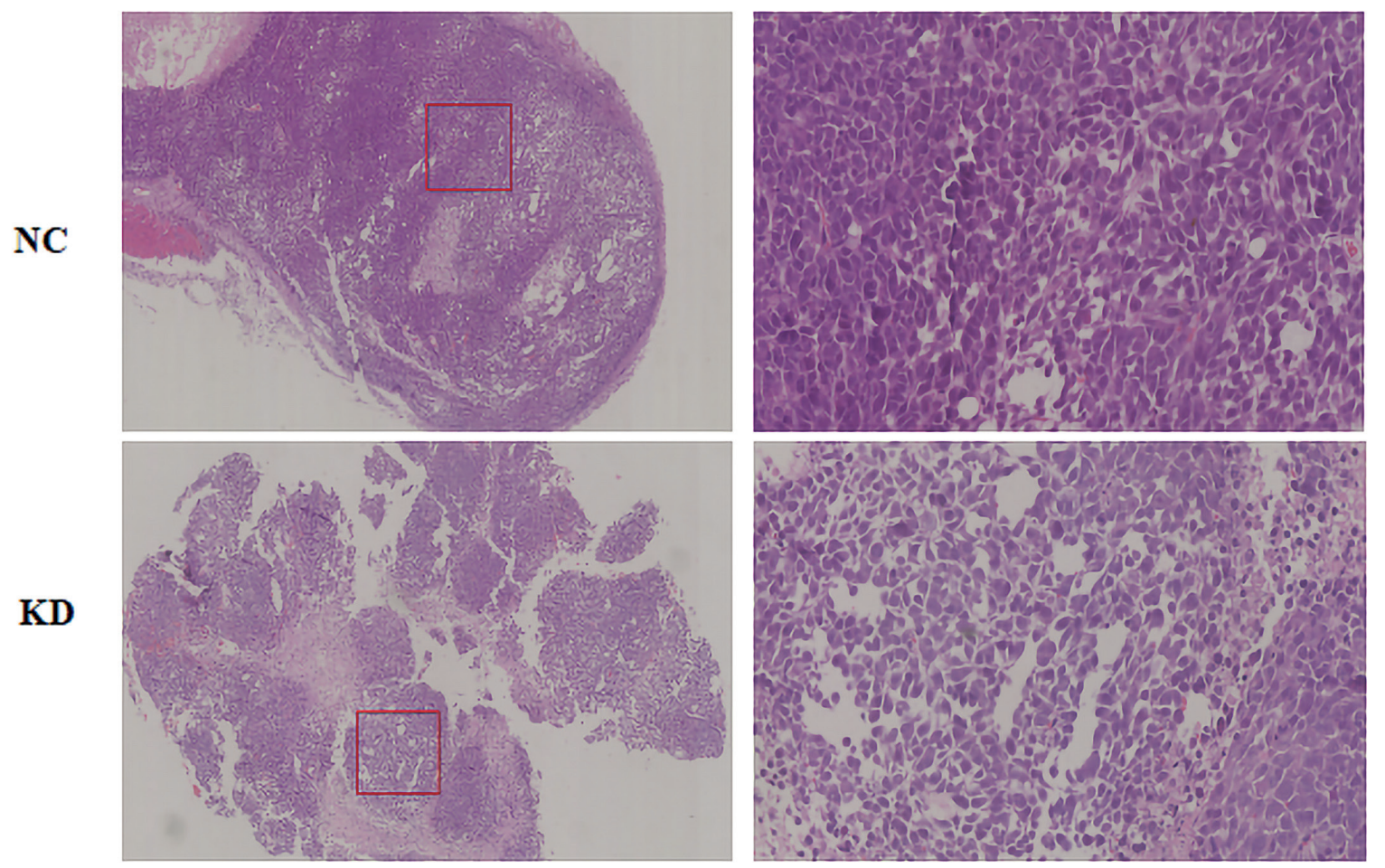

Fig. 2 HE staining of subcutaneous tumor tissue sections of nude mice (magnification, $\times 400$ ).

A

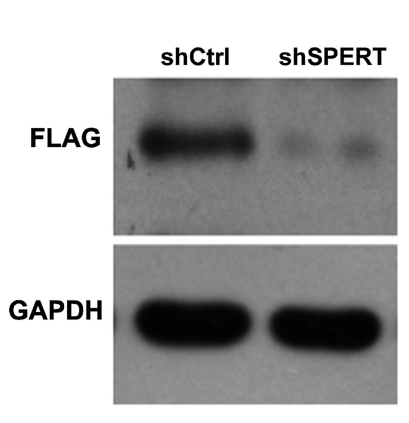

B

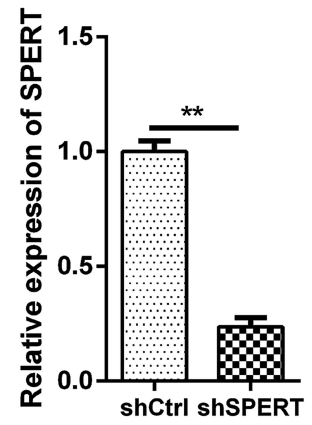

Fig. 3 Results of SPERT expression detection in subcutaneous tumor tissues of nude mice. Compared with the shCtrl group, the SPERT expression in the shSPERT group was significantly reduced. (A) Western blot analysis of SPERT protein. shCtrl, control group; shSPERT, SPERT knock-down group; $(B)$ the bar represents the relative gray values of the western blot $\left({ }^{\star *} P<0.01\right)$. GAPDH was used as a loading control.

TUNEL assay was used to detect the apoptosis of cells in the subcutaneous tumorigenesis in the KD group and $\mathrm{NC}$ group. The experimental results were as follows: the apoptosis index of cells in the $\mathrm{KD}$ group was $53.6 \pm 7.8 \%$, and that of the $\mathrm{NC}$ group was $10.5 \pm 2.2 \%$, with significant differences $(P<$ $0.05)$. The apoptosis index of tumor cells in the KD

group was higher (Fig. 5), indicating that SPERT knockdown can increase the apoptosis of tumor cells in the KD group. Through immunohistochemical detection of Ki-67 expression in tumor tissues and TUNEL assay of cell apoptosis, we found that the proliferation activity of tumor cells in tumor tissues formed after SPERT knockdown was reduced and cell apoptosis was increased, which also indicated that SPERT silencing could inhibit tumor growth in vivo.

The influence of SPERT silencing on the p38MAPK/ HSP27 signaling pathway

The expression changes of p38MAPK, HSP27, caspase- 3 and PARP (poly ADP-ribose polymerase) in the p38MAPK/HSP27 signaling pathway were further analyzed by Western blot (WB). These factors play an important role in the process of apoptosis. WB results indicated that the phosphorylation levels of p38MAPK and HSP27 protein in the KD group (shSPERT group) were higher than those in the NC group (shCtrl group) (Figs. 6 and 7), and the expression of PARP and caspase-3 protein was also increased (Figs. 8 and 9). There were statistical differences between the two groups $(P<0.01)$. The experimental results showed that the expression of these signaling molecules increased after SPERT 

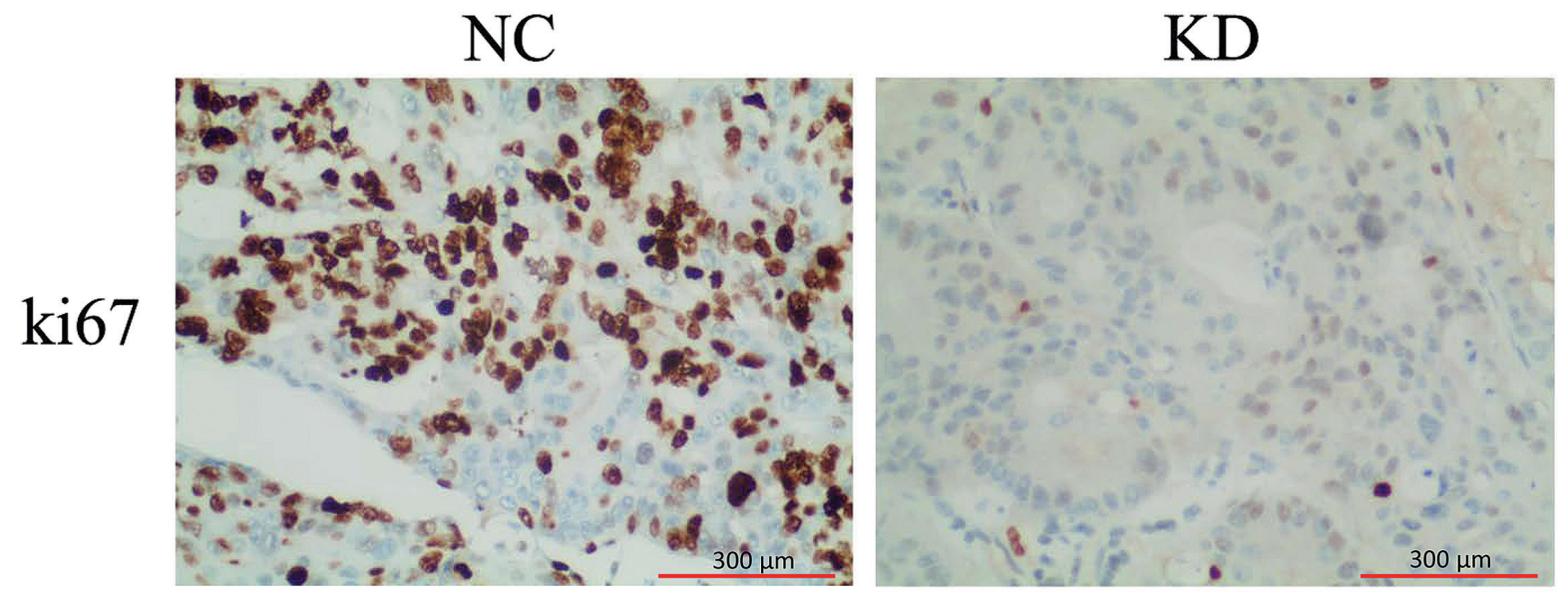

Fig. 4 Immunohistochemical staining for detection of Ki67 expression in tumor tissues (magnification, $\times 400$ ). The expression of Ki67 in tumor tissues of NC group was significantly higher than that of KD group, and the proliferation activity of cells was stronger.
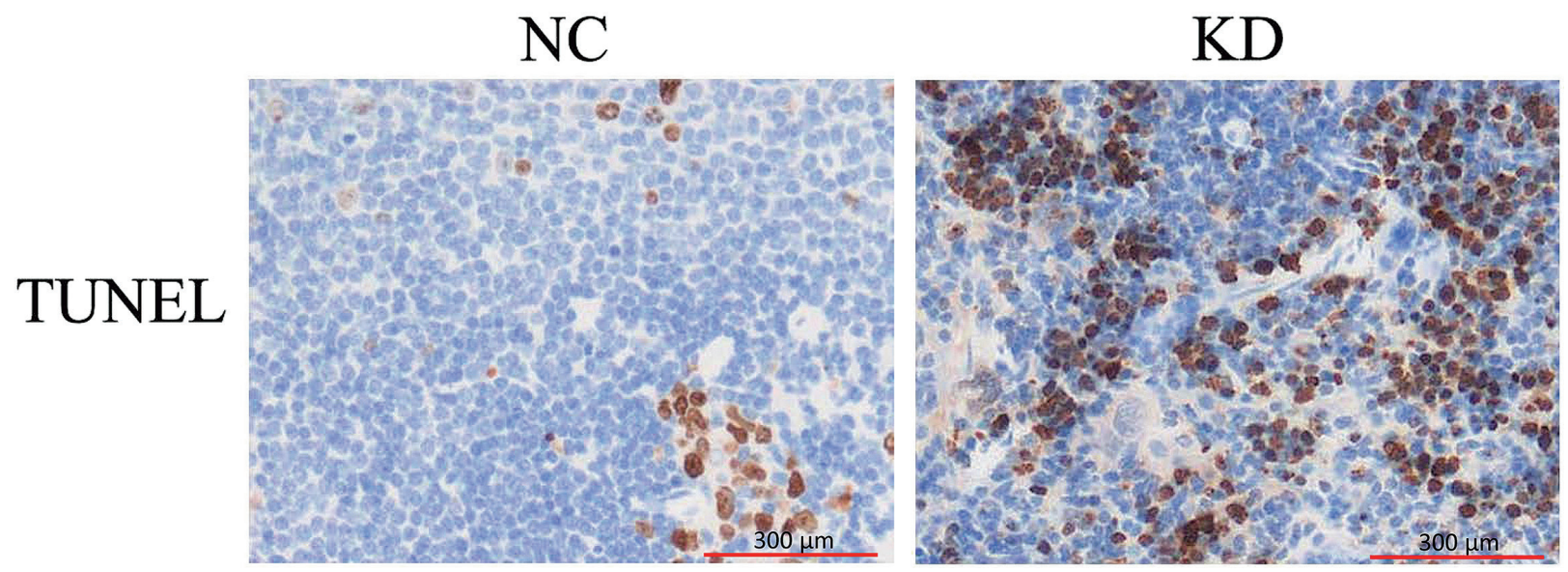

Fig. 5 TUNEL assay detects cell apoptosis in tumor tissues (magnification, $\times 400$ ). The apoptosis of tumor tissues in the KD group was significantly higher than that in the NC group.

silencing, indicating that SPERT silencing may promote apoptosis of RKO cells by affecting the p38MAPK/HSP27 signaling pathway.

\section{DISCUSSION}

Colorectal cancer is a genetically heterogeneous disease, which causes the transformation of normal mucosa into adenoma and then into carcinoma through complex interactions of multiple genes and pathways. Understanding the genetic mutations and their molecular mechanisms related to the occurrence of colorectal cancer can help to identify new therapeutic targets and develop strategies for the treatment of colorectal cancer, which have important effects on the treatment and prognosis of CRC. With the in-depth research of gene function and molecular mechanism, more and more evidence shows that MAPK signaling pathway not only plays an important role in regulating the proliferation and apoptosis of colorectal cancer cells (Morrison 2012). It is also involved in cell adhesion, tumor angiogenesis, tumor invasion and distant metastasis in colorectal cancer (Fang and Richardson 2005).

In the previous cell experiments, we found that SPERT silencing can inhibit the proliferation of human CRC RKO cells and promote cell apoptosis, and preliminary studies showed that it may act through the p38MAPK/HSP27 signaling pathway (Zheng and Chen 2018). Animal experiments are more convincing because they can simulate human physiological and pathological conditions better than 


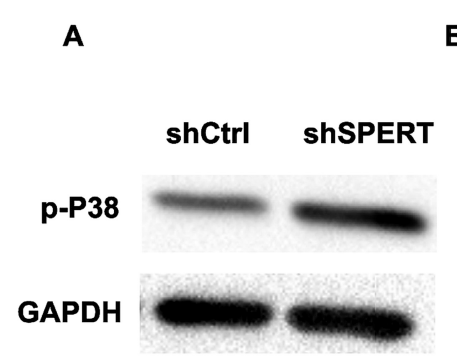

Fig. 6 The phosphorylation level of p38 MAPK protein increased significantly after SPERT knockdown. (A) Western blot analysis of p-P38 protein. shCtrl, control group; shSPERT, SPERT knock-down group; (B) the bar represents the relative gray values of the western blot $\left({ }^{\star *} P<0.01\right)$. GAPDH was used as a loading control.

cell culture experiments. In this experiment, BALB/c mice were selected as experimental subjects to explore the effect of SPERT silencing on tumor growth in animals to observe the changes in tumor volume and weight in mice, and further verify the function of SPERT from animal model experiments. Experimental data showed that the volume and weight of subcutaneous tumors in the SPERT silencing group were significantly lower than those in the control group. Meanwhile, immunohistochemical HE staining showed that the subcutaneous tumors in the two groups of nude mice were malignant tumors, indicating the successful construction of our transplanted tumor model. Western blot results also showed that the expression level of SPERT in the KD group was significantly lower than that in the NC group, further confirming that reduced SPERT level could lead to reduced tumor burden, and SPERT silencing could inhibit the growth of subcutaneous tumors in nude mice.

Ki67 was first discovered and defined by Gerdes et al. (1983) when he studied the immunostaining of mitotic cells, which showed that the expression of Ki67 was closely related to the proliferative activity of cells and was widely used as a marker of tumor cell proliferation (Scholzen and Gerdes 2000). In order to further confirm our in vivo study, we determined the proliferative activity and apoptosis of tumor cells in transplanted tumors by detecting the expression of Ki67 and the TUNEL assay for detecting apoptosis in tumor tissues. We found that the proliferative activity of tumor cells reduced and cell apoptosis increased after SPERT knockdown. The experimental data also indicated that SPERT silencing could inhibit tumor growth in animals.

p38MAPK was first called HOG 1, which was

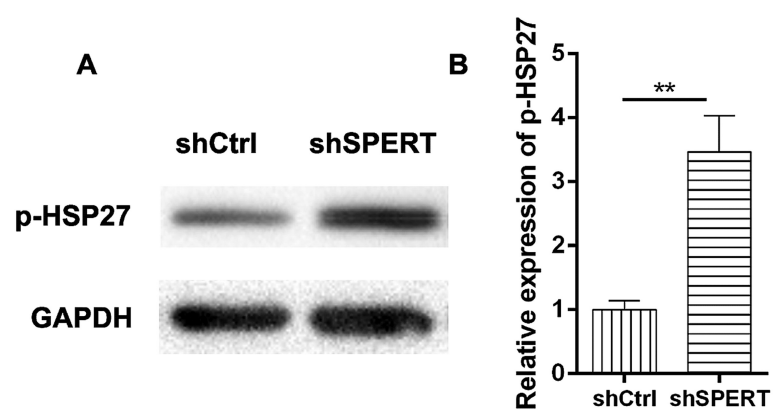

Fig. 7 The phosphorylation level of HSP27 protein increased significantly after SPERT knockdown. (A) Western blot analysis of HSP27 protein. shCtrl, control group; shSPERT, SPERT knock-down group; (B) the bar represents the relative gray values of the western blot $\left({ }^{\star \star} P<0.01\right)$. GAPDH was used as a loading control.

discovered by Brewster et al. (1993) when studying the changes of osmotic pressure in the extracellular environment. Subsequently, Han et al. (1994) identified a $38 \mathrm{kDa}$ polypeptide and named it $\mathrm{p} 38$ aMAPK (p38MAPK). This study has shown that the p38MAPK pathway plays an important role in Alzheimer's disease, cardiovascular dysfunction, inflammation, and cancer. Our experimental results in this part show that the phosphorylation level of p38MAPK is increased, indicating that $\mathrm{p} 38$ plays a positive role in inhibiting proliferation and promoting apoptosis of human CRC RKO cells, which is consistent with other reports (Cuenda and Rousseau 2007). The substrates of p38 include p53, STAT-1, Cdc25, Tau, HSP27, TAB, ATF2, CREB and other key molecules. In the preliminary screening experiment, we found that the phosphorylation level of HSP27 was increased. There are three phosphorylation sites of HSP27: Ser-15, Ser-78 and Ser-82. The phosphorylation of Ser-82 is essential in the process of reducing HSP27 complex to a small oligomer. The phosphorylation of Ser-15 can regulate the interaction between HSP27 and proteins such as protein kinase B, F-actin or granzyme A. The third serine residue (Ser-78) in human HSP27 is poorly conserved, and its role in regulating the structure of HSP27 remains unclear (Lambert et al. 1999). It is known that large oligomers are the structural form necessary for anti-apoptotic activity of HSP27. Therefore, the expression level of HSP27 is low and mainly exists in the form of large oligomers in the normal state of cells. HSP27 in this polymerization state is dephosphorylated and generally inactive. When cells are stimulated by certain factors, HSP27 expression is increased and phosphorylated, and large oligomers are depolymerized to form small oligomers (Bruey 


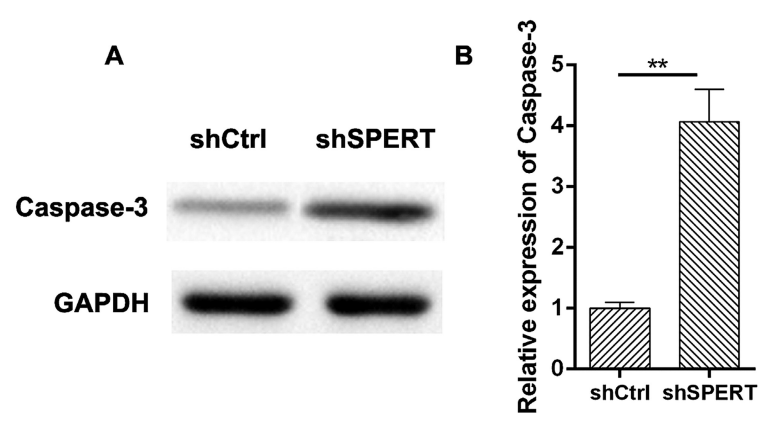

Fig. 8 The expression level of Caspase-3 protein increased significantly after SPERT knockdown. (A) Western blot analysis of Caspase-3 protein. shCtrl, control group, shSPERT, SPERT knock-down group; (B) the bar represents the relative gray values of the western blot $\left({ }^{\star \star} P<0.01\right)$. GAPDH was used as a loading control.

et al. 2000). Currently, there are three main phosphorylation sites for HSP27, and they have antibodies for phosphorylation of their respective sites. Our experiment considered that the anti-apoptotic effect of HSP27 is mainly exerted through its large oligomeric form, and the phosphorylation of Ser-82 site plays an important role in the conversion of HSP27 large and small oligomers, so we chose monoclonal antibody against Ser-82 phosphorylation (pSer82) for this experiment. Phosphorylated HSP27 regulates cell apoptosis through the following two pathways: (1) It can inhibit the binding of DAXX (Death domain-associated protein) to Fas, thus affecting the process of FAS-mediated apoptosis and inhibiting cell apoptosis; (2) After phosphorylation of HSP27, large oligomers are dissociated to form small oligomers, which lose the inhibition effect on mitochondria-mediated apoptosis and produce pro-apoptotic effect. In this experiment, SPERT silencing can promote the apoptosis of human CRC RKO cell, and its mechanism should be the result of the interaction of the above two signaling pathways, and it should be dominated by the second pathway. In other words, after the phosphorylation of HSP27, the balance between HSP27 small oligomers and large oligomers was transferred to small oligomers, that is, after the dissociation of large oligomers, the inhibition of mitochondrial pathway-mediated apoptosis was lost, thus producing pro-apoptotic effect.

Caspase-3, also known as CPP32, was discovered by Fernandes-Alnemri et al. in 1994 and belongs to the cysteine aspartate protease family. It plays a key role in the regulation of cell apoptosis by protease cascade mediated by members of the caspase family and is an executioner caspase involved in cell apoptosis (Fernandes-Alnemri et al. 1994). Caspase-3 is

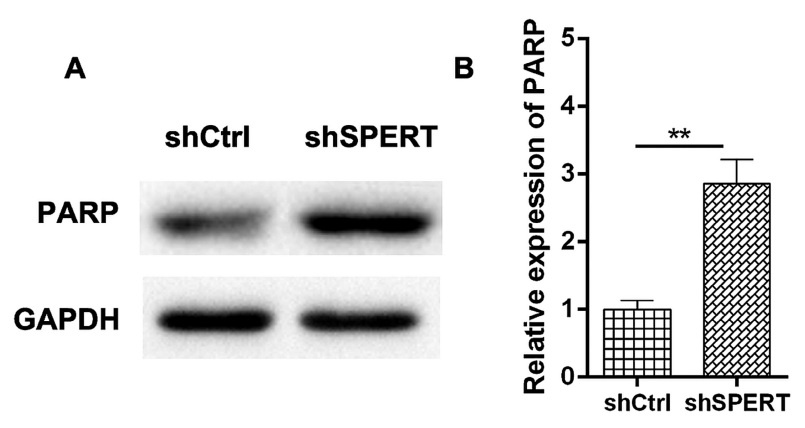

Fig. 9 The expression level of PARP protein increased significantly after SPERT knockdown. (A) Western blot analysis of PARP protein. shCtrl, control group; shSPERT, SPERT knock-down group; (B) the bar represents the relative gray values of the western blot $\left({ }^{\star \star} P<0.01\right)$. GAPDH was used as a loading control.

initially present as an inactive monomer protease, which needs to be activated by dimerization and cleavage, playing the ultimate executor role in both endogenous and exogenous pathways of apoptosis. The exogenous apoptotic pathway is activated by binding of ligands to death receptors, which, with the help of adaptor proteins (FADD/Tradd), recruits caspase- 8 and activates it by dimerization, and activated caspase- 8 then activates caspase- 3 by cleavage, thus initiating apoptosis. The endogenous or mitochondrial apoptotic pathway can be activated by various cellular stresses, leading to the release of cytochrome $\mathrm{C}$ from mitochondria and the formation of apoptotic bodies composed of APAF 1, cytochrome C, ATP and caspase-9, thus activating caspase-9, and activated caspase- 9 then initiates apoptosis by cutting and activating caspase-3 (Mcllwain et al. 2013). We found that the expression of caspase- 3 was significantly increased in this experiment, and it is speculated that the phosphorylation of HSP27 lost its inhibitory effect on mitochondria-mediated apoptosis, which led to the activation of caspase-3 downstream of its signaling pathway and promoted cell apoptosis. PARP is an enzyme family that catalyzes the transfer of ADP-ribose from NAD + to protein substrates, and is the most important substrate for caspase-3 (Leung 2017). PARP regulates the function of target proteins through ADP-ribose modification, which is a unique post-translational modification and plays an important role in biological activities (Liu and Yu 2015; Bock and Chang 2016). Relevant studies have shown that large-scale activation of PARP can cause depletion of NAD+ and ATP, leading to cell death, so it is speculated that activation of PARP is an essential step in the process of apoptosis (Scovassi and Poirier 1999; Ivana and Diederich 
2004). Our experimental results showed that human RKO cells showed proliferation-inhibiting and apoptosis-promoting effects after SPERT silencing, and further molecular studies revealed elevated expression of caspase- 3 and PARP. The experimental results also confirmed that caspase-3 played an executor role in the process of apoptosis, and the increased expression of PARP also explained its role as an important indicator of caspase-3 activation.

In summary, according to the results of this experiment, we describe a molecular pathway that promotes apoptosis of human CRC RKO cell after SPERT silencing: the p38MAPK/HSP27 signaling pathway. In the first step, SPERT silencing stimulates the stress response of cells, leading to an increase in the phosphorylation level of p38MAPK. Second, the activated p38MAPK further phosphorylates the downstream substrates HSP27. When HSP27 is phosphorylated (Ser-82 site), HSP, which originally exists as a large oligomer, depolymerizes to form a small oligomer. Third, the small oligomer formed after the dissociation of the large oligomer HSP27 loses its inhibitory effect on mitochondria-mediated apoptosis, leading to the activation of caspase- 3 downstream of its signaling pathway, and the increase of PARP expression, which promotes cell apoptosis.

\section{Acknowledgements}

The present study was supported in part by a grant from the Natural Science Foundation of Fujian Province (No. 2019J01582).

\section{CONFLICT OF INTEREST}

The authors declare no conflict of interest.

\section{REFERENCES}

Bock FJ and Chang P (2016) New directions in poly(ADPribose) polymerase biology. FEBS $J \mathbf{2 8 3}, 4017-4031$.

Bray F, Ferlay J, Soerjomataram I, Siegel RL, Torre LA, et al. (2018) Global cancer statistics 2018: GLOBOCAN estimates of incidence and mortality worldwide for 36 cancers in 185 countries. CA Cancer J Clin 68, 394-424.

Brenner H, Kloor M and Pox CP (2014) Colorectal cancer. Lancet 383, 1490-1502.

Brewster JL, de Valoir T, Dwyer ND, Winter E and Gustin MC (1993) An osmosensing signal transduction pathway in yeast. Science 259, 1760-1763.

Bruey JM, Paul C, Fromentin A, Hilpert S, Arrigo AP, et al. (2000) Differential regulation of HSP27 oligomerization in tumor cells grown in vitro and in vivo. Oncogene 19, 4855-
4863.

Chery J (2016) RNA therapeutics: RNAi and antisense mechanisms and clinical applications. Postdoc J 4, 35-50.

Cuenda A and Rousseau S (2007) p38 MAP-kinases pathway regulation, function and role in human diseases. Biochim Biophys Acta 1773, 1358-1375.

Fang JY and Richardson BC (2005) The MAPK signaling pathways and colorectal cancer. Lancet Oncol 6, 322-327.

Fernandes-Alnemri T, Litwack G and Alnemri ES (1994) CPP32, a novel human apoptotic protein with homology to Caenorhabditis elegans cell death protein Ced-3 and mammalian interleukin-1 betaconverting enzyme. $J$ Biol Chem 269, 30761-30764.

Gerdes J, Schwab U, Lemke H and Stein H (1983) Production of a mouse monoclonal antibody reactive with a human nuclear antigen associated with cell proliferation. Int J Cancer 31, $13-20$.

Han J, Lee JD, Bibbs L and Ulevitch RJ (1994) A MAP kinase targeted by endotoxin and hyperosmolarity in mammalian cells. Science 265, 808-811.

Ivana Scovassi A and Diederich M (2004) Modulation of poly(ADP-ribosylation) in apoptotic cells. Biochem Pharmacol 68, 1041-1047.

Lambert H, Charette SJ, Bernier AF, Guimond A and Landry J (1999) HSP27 multimerization mediated by phosphorylation-sensitive intermolecular interactions at the amino terminus. J Biol Chem 274, 9378-9385.

Leung AKL (2017) PARPs. Curr Biol 27, R1256-R1258.

Liu C and Yu X (2015) ADP-ribosyltransferases and poly ADPribosylation. Curr Protein Pept Sci 16, 491-501.

Mcllwain DR, Berger T and Mak TW (2013) Caspase functions in cell death and disease. Cold Spring Harb Perspect Biol 5, a008656.

Morrison DK (2012) MAP kinase pathways. Cold Spring Harb Perspect Biol 4, a011254.

Offit K (2016) The future of clinical cancer genomics. Semin Oncol 43, 615-622.

Sakatani T and Onyango P (2003) Oncogenomics: prospects for the future. Expert Rev Anticancer Ther 3, 891-901.

Scholzen T and Gerdes J (2000) The Ki-67 protein: From the known and the unknown. J Cell Physiol 182, 311-322.

Scovassi AI and Poirier GG (1999) Poly(ADP-ribosylation) and apoptosis. Mol Cell Biochem 199, 125-137.

Strausberg RL, Simpson AJ, Old LJ and Riggins GJ (2004) Oncogenomics and the development of new cancer therapies. Nature 429, 469-474.

Torre LA, Bray F, Siegel RL, Ferlay J, Lortet-Tieulent J, et al. (2015) Global cancer statistics, 2012. CA Cancer J Clin 65, 87-108.

Wang Y, Cao W, Yu Z and Liu Z (2009) Downregulation of a mitochondria associated protein SLP-2 inhibits tumor cell motility, proliferation and enhances cell sensitivity to chemotherapeutic reagents. Cancer Biol Ther 8, 1651-1658.

Yang G, Wheeler TM, Kattan MW, Scardino PT and Thompson TC (1996) Perineural invasion of prostate carcinoma cells is associated with reduced apoptotic index. Cancer 78, 12671271.

Zheng LZ and Chen SZ (2018) shRNA-induced knockdown of the SPERT gene inhibits proliferation and promotes apoptosis of human colorectal cancer RKO cells. Oncol Rep 40, 813-822. 